\title{
Choques de Oferta em Modelos de Metas Inflacionárias*
}

\author{
Fabio Kanczuk
}

Sumário: 1. Introdução; 2. Modelo; 3. Calibração; 4. Simulações e resultados: segundos momentos das séries; 5. Modelo estrutural e choques de oferta; 6 . Pseudo-dólar; 7. Simulações e resultados: modelo estrutural; 8. Conclusões

Palavras-chave: ciclos reais brasileiros; contratos salariais; moeda.

Códigos JEL: E32; E52.

Construímos um modelo de equilíbrio geral dinâmico, calibrado para a economia brasileira, em que uma fração das firmas fixa os salários nominais com um período de avanço, de acordo com o seu valor esperado. As séries geradas através de simulações nessa economia artificial são consistentes com os dados reais, e com uma estimação típica de um modelo estrutural de Metas Inflacionárias. Argumentamos que, por não considerar a presença de choques de oferta, esses modelos estruturais estão especificados incorretamente. Em contraste, nosso modelo pode separar os efeitos dos choques de oferta e demanda, além de ser (potencialmente) robusto a Crítica de Lucas.

We construct a dynamic general equilibrium model calibrated to the Brazilian economy, in which a fraction of the firms set nominal wages one period in advance, according to its expected value. The artificial economy simulations generate series consistent with real data, and with a typical estimation of a structural Inflation Targeting model. We argue that these structural models specifications are incorrect, for not considering supply shocks. In contrast, our model can separate supply and demand shocks effects, in addition of being (potentially) robust to the Lucas' Critique.

\section{Introdução}

A recente mudança na forma de atuação do Banco Central do Brasil (BACEN), com a adoção de um regime de Metas Inflacionárias, fez aparecer uma demanda por modelos econômicos que possam servir como suporte e auxílio na tomada de decisões de política monetária (Fraga, 2000). O mais usado desses modelos, denominado pelo BACEN de "modelo estrutural de pequeno porte", é formado

*Artigo recebido em dez. 2002 e aprovado em out. 2003. 
por estimações econométricas das principais relações entre as variáveis macroeconômicas. Nesse artigo argumentamos que esse procedimento não somente sofre de falta de robustez a Crítica de Lucas, mas também falha em não considerar choques de oferta.

Para tal, desenvolvemos um modelo de equilíbrio geral dinâmico, em linha com os modelos de ciclos reais, mas adaptado para o estudo de políticas monetárias alternativas. Tomamos como base o modelo de Kanczuk (2002a), em que as firmas estão sujeitas a restrições de capital de giro, e assumimos um tipo de preferências que é consistente com os fatos estilizados das oscilações da economia brasileira (Kanczuk, 2001, 2002b). Contudo, em vez de utilizar o modelo para estudar as componentes cíclicas da economia, desenvolvemos um setor monetário adequado a simulações.

Em particular, introduzimos moeda e rigidez salarial, de forma semelhante a Cooley e Hansen (1998). Assim como esses autores, assumimos que uma parte das firmas paga salários nominais que foram contractuados no período anterior, mas de acordo com seu valor esperado para o período seguinte. Esta hipótese segue a literatura de contratos de trabalho (Blinder e Mankiw, 1984), e pode ser justificada em termos de "contratos implícitos" , que aparecem devido à assimetria de informação entre as firmas e os trabalhadores.

Quando nossa economia artificial é calibrada para o Brasil pós Plano Real, que é o período utilizado para as estimações do BACEN, suas simulações são consistentes com os segundos momentos dos dados de fato observados. Além disso, estimações do modelo estrutural nesses dados artificialmente gerados implicam em coeficientes próximos aos estimados sobre os dados reais.

Uma análise específica da lógica econômica que baliza o modelo estrutural do BACEN mostra que o mesmo é consistente somente com choques de demanda. Contudo, os dados reais parecem ser conseqüência da existência simultânea de choques de oferta e demanda. Isso implica em que o modelo usualmente estimado tem especificação incompleta, sofrendo dessa falta de identificação. Em contraste, nosso modelo pode separar os dois tipos de choque, estimando corretamente as relações.

A seguir, utilizamos o modelo para mostrar como a conhecida Crítica de Lucas (Lucas, 1976) também constitui um problema da utilização direta de econometria nesses modelos. Para tal alteramos a política do governo e mostramos que os coeficientes estimados também se alteram. Com esse experimento, sugerimos como modelos com microfundamentos podem e devem ser utilizados por bancos centrais cada vez que uma decisão sobre política monetária deve ser feita.

O trabalho está dividido da seguinte forma. A próxima seção desenvolve o 
modelo. A seção três explicita os dados e a calibração. A seção quatro mostra os resultados das simulações das economias artificiais, e os compara com os segundos momentos dos dados reais. A seção cinco discute a hipótese no modelo estrutural, em especial seu problema de especificação por não incorporar choques de oferta. A seção seis introduz um preço artificial, que denominamos de "pseudo-dólar", para que nosso modelo possa, ainda que de forma ad-hoc, gerar séries de depreciação cambial. A seção sete contrasta as estimações sobre os dados artificiais com a sobre os dados reais, verifica o problema de identificação e da robustez a Crítica de Lucas. Uma última seção conclui.

\section{Modelo}

Nossa economia artificial é povoada por um continuum de famílias idênticas e de vida infinita, com nomes no intervalo $[0,1]$. Cada uma dessas famílias tem uma dotação de tempo para cada período que deve ser repartida entre lazer $\left(l_{t}\right)$ e trabalho $\left(h_{t}\right)$. A dotação de tempo é normalizada para uma unidade, isto é, $h_{t}+l_{t}=1$. Adicionalmente, as famílias possuem um nível inicial de capital $k_{o}$ que elas alugam para as firmas, e podem aumentar através de investimentos, e um nível inicial de moeda $m_{0}$ que não recebe nenhuma remuneração. A razão para que moeda não seja completamente dominada pela existência de capital reside na presença da moeda na função de utilidade.

A utilidade das famílias para cada período é definida em termos das seqüências estocásticas de consumo, moeda real e lazer:

$$
U_{s}=E_{s} \sum_{t=s}^{\infty} \beta^{t-s} u\left(c_{t}, m_{t+1} / p_{t}, h_{t}\right)
$$

em que:

$c_{t}, h_{t}$ e $m_{t+1}$ representam seqüências Arrow-Debreu de consumo, trabalho e moeda contingentes ao estado;

$p_{t}$ representa o preço do bem final, também contingente ao estado, e; $[0,1]$ é um parâmetro de desconto. ${ }^{1}$

Assim como Kanczuk (2001, 2002b), utilizamos a forma funcional para a utilidade instantânea semelhante à proposta por Greenwood et alii (1988), a qual tem a propriedade de que a elasticidade intertemporal de substituição associada com o lazer é zero:

\footnotetext{
${ }^{1}$ Como se sabe, a formulação de moeda na função de utilidade deve ser vista como uma forma reduzida de uma outra formulação, em que o papel da moeda é melhor especificado. Vide, por exemplo, Correia e Teles (2000).
} 


$$
u\left(c_{t}, m_{t+1} / p_{t}, h_{t}\right)=\log \left[c_{t}^{\omega}\left(m_{t+1} / p_{t}\right)^{1-\omega}-v_{1} h_{t}^{v_{2}}\right]
$$

em que $\omega \in[0,1], v_{1}>0$ e $v_{2}>1$. Para que economias com essas preferências sejam consistentes com um estado estacionário em que há crescimento, é necessário que a desutilidade de trabalhar no mercado aumente com o nível de progresso tecnológico. Este efeito pode ser interpretado como progresso tecnológico associado com a produção em atividades domésticas (veja nota de rodapé 11 em Christiano et alii (1997)). A utilização dessa forma funcional para as preferências é essencial para que se obtenha um caráter contra-cíclico para exportações líquidas, conforme nos mostram os trabalhos de Correia et alii (1995) e Kanczuk (2001). Embora não tenhamos comércio exterior neste modelo, a escolha dessa preferência torna o modelo compatível com essa propriedade.

As famílias que povoam a economia ofertam trabalho e capital às firmas que têm acesso a uma tecnologia descrita por uma função de produção Cobb-Douglas:

$$
Y_{t}^{\prime}=F\left(z_{t}, K_{t}, H_{t}\right)=\exp \left(z_{t}\right) K_{t}^{\theta} H_{t}^{1-\theta}
$$

em que:

trabalho $\left(H_{t}\right)$ e capital acumulado $\left(K_{t}\right)$ são insumos, e;

$z_{t}$ é um parâmetro estocástico de produtividade. Assumimos que $z_{t}$ evolui de acordo com um processo autoregressivo, com lei de formação:

$$
z_{t}=\rho_{z} z_{t-1}+\epsilon_{z t}
$$

em que $\epsilon_{z}$ é distribuídos de acordo com uma normal, com média zero e desvios padrão $\sigma_{\epsilon}$

O capital deprecia exponencialmente a uma taxa $\delta$ e os consumidores adicionam ao estoque de capital através de investimentos de uma parte do produto a cada período. Investimentos em $t$ produzem capital em $t+1$, de forma que a lei de formação para o capital agregado é

$$
K_{t+1}=(1-\delta) K_{t}+X_{t}
$$

em que $X_{t}$ representa o investimento (ou formação bruta de capital).

As firmas alugam capital e contratam trabalho. Em contraste com os modelos mais usuais de ciclos reais, assumimos, como em Kanczuk (2002a) que as firmas também têm que permanecer, em cada período, com uma fração da produção, na forma de capital de giro. Essa restrição ocorre devido à falta de sincronismo entre as receitas e despesas, e faz com que as firmas permaneçam com os recursos 
necessários para pagar por parte dos salários e do aluguel do capital em caixa. Note que essa restrição é análoga a uma de cash-in-advance, mas ocorre no lado da produção.

Denotamos por $Q_{t}$ a quantidade de capital de giro entre $t$ e $(t+1)$. Esses recursos são emprestados pelo governo no início de cada período, e devolvidos no final do período sem receberem remuneração. Alternativamente, pode-se pensar que as firmas tomam esses empréstimos de intermediários financeiros, mas como no nosso modelo o governo transfere recursos de volta às famílias na forma lumpsum, e seria exatamente isso que intermediários financeiros fariam com seus lucros, esta hipótese é inofensiva. Podemos escrever o problema da firma como

$$
\operatorname{Max}\left\{Y_{t}-w_{t} H_{t}-u_{t} K_{t}-\left(Q_{t} / p_{t}\right)+\frac{\left(Q_{t} / p_{t+1}\right)}{\left(1+r_{t}\right)}\right\}
$$

sujeito às restrições de capital de giro,

$$
Q_{t} / p_{t} \geq \alpha Y_{t}
$$

em que:

$w$ e $u$ correspondem aos produtos marginais do trabalho e capital;

$r$ corresponde aos juros reais, e;

$\alpha$ é uma constante. Note que como o capital de giro não rende juros, essa desigualdade sempre será restritiva (binding), e que para $\alpha=0$ o problema da firma se reduz ao caso usual.

As famílias, em contraste com as firmas, resolvem um problema intertemporal, e têm que formar expectativas sobre preços futuros. Elas escolhem consumo, investimento, demanda por (novos) títulos externos, moeda e horas de trabalho em cada data para maximizar o valor descontado da utilidade sujeito às seqüências de restrições orçamentárias e leis de formação de capital e títulos:

$$
\begin{gathered}
c_{t}+x_{t}\left[1+\phi\left(x_{t} / k_{t}\right)\right]+m_{t+1} / p_{t} \leq w_{t} h_{t}+u_{t} k_{t}+m_{t} / p_{t}+T_{t} \\
k_{t+1}=(1-\delta) k_{t}+x_{t}
\end{gathered}
$$

em que:

$T_{t}$ corresponde a transferências lump-sum e a função $\phi_{1}\left(x_{t} / k_{t}\right)$ representa os custos associados com a instalação de capital. Como usual, assumimos que sua forma funcional é $\phi_{1}\left(x_{t} / k_{t}\right)=\phi\left(x_{t} / k_{t}\right)^{2}$, em que, com leve abuso de notação, o $\phi$ do lado direito da equação é uma constante. Essa formulação implica que os custos sejam zero no estado estacionário. 
A ação do setor público sobre o setor privado é distorcer as alocações através da emissão de moeda e da cobrança de impostos, e devolver esses recursos na forma lump-sum. O estoque nominal de moeda é determinado pela lei de formação

$$
M_{t+1}=e^{g_{t}} M_{t}
$$

em que $g$ representa um processo estocástico que será endogenamente determinado, através da taxa de juros nominal. No estado estacionário, esse processo tem média $g^{m}$. Com isso, os preços da economia têm uma tendência geométrica com razão $e^{g m}$, e são normalizados para as variáveis estacionárias $\hat{p}_{t}=p_{t} / M_{t}$ e $\hat{m}=m_{t} / M_{t}$. As transferências governamentais são dadas por,

$$
T_{t}=\left(M_{t} / p_{t}\right)\left[e^{g_{t}}-1\right]
$$

A forma do processo estocástico de $g$, por sua vez, é determinado pela evolução dos juros nominal, de acordo com a demanda por moeda (i.e., a equação de Euler para moeda). Este procedimento é o proposto por Christiano e Gust (2000) e Chari et alii (2000), entre outros. Consideramos que o governo escolhe a taxa primária $i_{t}$, mas que a taxa que efetivamente corresponde ao custo de oportunidade da moeda é dada por $\left(1-\tau_{i}\right) i_{t}$. O coeficiente $\tau_{i}$ captura tanto o efeito de impostos financeiros como a presença de risco dos títulos públicos.

Assim como Taylor (1999), assumimos que $i_{t}$ segue uma lei de formação da forma

$$
i_{t}=\left(1-\rho_{i}\right) i^{m}+\rho_{i}^{i_{t-1}}+\rho_{\pi}\left(\pi_{t-1}-\pi^{m}\right)+\rho_{y} y_{t-1}+\epsilon_{i t}
$$

em que:

$i^{m}$ e $\pi^{m}$ são constantes, refere-se a inflação;

$y$ refere-se ao hiato do produto (i.e. o logarítmo do produto filtrado por HodrickPrescott), e;

$\epsilon_{i}$ é distribuído de acordo com uma normal, com média zero e desvio padrão $\rho_{i}$.

De forma semelhante a Cooley e Hansen (1998) e Floden (2000), assumimos uma fração das firmas, que denotamos por $\mu$, pagam salários nominais que foram determinados um período atrás, de acordo com seu valor esperado. Isto é, o salário nominal contratado corresponde ao valor esperado do salário nominal que equilibra o mercado em uma economia sem contratos. A forma de solução envolve, primeiramente, resolver uma economia sem rigidez salarial. Como resultado dessa primeira parte, obtemos as funções de política de preço, horas trabalhadas e investimento, da forma: 


$$
\begin{gathered}
H_{t}=a_{0}+a_{1} z_{t}+a_{2} i_{t-1}+a_{3} \epsilon_{i t}+a_{4} \hat{p}_{t-2}+a_{5} \hat{p}_{t-1}+a_{6} y_{t}+a_{7} K_{t} \\
X_{t}=b_{0}+b_{1} z_{t}+b_{2} i_{t-1}+b_{3} \epsilon_{i t}+b_{4} \hat{p}_{t-2}+b_{5} \hat{p}_{t-1}+b_{6} y_{t}+b_{7} K_{t} \\
\hat{p}_{t}=c_{0}+c_{1} z_{t}+c_{2}^{i_{t-1}}+c_{3} \epsilon_{i t}+c_{4} \hat{p}_{t-2}+c_{5} \hat{p}_{t-1}+c_{6} y_{t}+c_{7} K_{t}
\end{gathered}
$$

A seguir, utiliza-se essas funções de política obtidas para alimentar a economia com contratos. O salário nominal nas firmas com rigidez é contratado para o valor esperado do produto marginal do trabalho:

$$
W^{c}=E\left\{p(1-\theta) e^{z}\left(K / H^{e}\right)^{\theta}\right\}
$$

em que $H^{e}$ é o valor esperado da demanda por trabalho. Após os choques tenham sido revelados, estas firmas decidem a demanda por trabalho de forma a maximizar lucro. Como $W^{c}$ está dado, elas escolhem o número de horas a serem trabalhadas tal que o produto marginal do trabalho seja igual ao salário real, isto é, elas escolhem $H^{c}$ tal que

$$
W / p=(1-\theta) e^{z}\left(K / H^{c}\right)^{\theta}
$$

Combinando estas duas equações, relembrando a normalização dos preços, e aproximando as expressões, obtém-se

$$
\log \left(H^{c}\right)=[E \log (H)]+(1 / \theta)\{\log \hat{p}-E[\log \hat{p}]+z-E[z]+g-E[g]\}
$$

Para preservar famílias idênticas, assumimos que elas oferecem trabalho simultaneamente para as firmas com e sem rigidez salarial. Assim, as horas trabalhadas em equilíbrio, $H^{*}$ correspondem à média das horas trabalhadas na economia como um todo, isto é,

$$
H^{*}=\mu H^{c}+(1-\mu) H
$$

Vale notar que, como os valores esperados das funções de política dependem das variáveis de estado defasadas, a equação de Bellman para economia com contratos inclui os valores presentes e passados das variáveis de estado. No mais, sua formulação é análoga àquelas, por exemplo, em Cooley (1995). 


\section{Calibração}

Utilizamos os dados disponíveis do sistema IPEADATA (www.ipeadata.gov.br) de 1980:1 a 2001:4. Mas, como estamos particularmente interessados no período de preços estáveis, focalizamos nossa atenção no período 1994:3 a 2001:4, após o plano Real.

A série de PIB corresponde ao "PIB a preços de mercado com índice encadeado", o qual é dessazonalizado através do método de fatores multiplicativos. A série de investimento é construída a partir da "taxa de investimento (\% PIB) a preços de 1990", e do PIB dessazonalizado. Como não dispomos de uma série de bens duráveis, os mesmos estão incluídos na série de consumo, ao invés de serem adicionados aos investimentos, prática usual em estudos da economia dos EUA. A série de consumo do governo também está adicionada á série de consumo. Para que possamos comparar essa série de consumo com os dados gerados por nossas economias artificiais, tomamos o cuidado de somar o consumo do governo ao consumo, em nossas simulações.

Como medida de inflação utilizamos o IPCA "centrado", como juros nominais a taxa SELIC, e como juros reais a diferença desses. Finalmente, como conceito de moeda, utilizamos M1.

A nossa calibração segue de perto o procedimento de Kanczuk (2002b), mas leva em conta o fato de nosso modelo não considerar crescimento tecnológico e populacional. Isto faz com que alguns dos parâmetros tenham que ser recalibrados.

Usando as médias no período para a inflação e para os juros nominais, obte$\operatorname{mos} g^{m}=2,4 \%$ e $i^{m}=6,7 \%$ (trimestrais). Para obtermos o valor da taxa de depreciação, utilizamos uma relação capital-trabalho anual de 3,0 (em acordo com a obtida por Araujo e Ferreira (1999)), e que a taxa média de investimento no período foi de $20 \%$. A lei de formação de capital no estado estacionário é

$$
(i / y)=\delta(k / y)
$$

o que implica em $\delta=1,7 \%$ (trimestral). Seguindo Kanczuk (2002a), utilizamos $\theta=0,40$, que está em linha com Gollin (2002). A equação de Euler para o investimento no estado estacionário, e a expressão para a remuneração do capital implicam em

$$
1=\beta[\theta(y / k)+1-\delta]
$$

o que determina o parâmetro de desconto intertemporal $\beta=0,98$. A equação de Euler sobre títulos do governo (que não existem, sem perda de generalidade) implica em $1=\beta\left(1+i^{m}\left(1-\tau_{i}\right)\right)$, determinando $\tau_{i}=39 \%$. 
Para calibrarmos utilizamos a curva de demanda por moeda, obtida a partir das condições de primeira ordem para o consumo e para moeda, dada por

$$
\frac{(M / p)}{c} \frac{\omega}{1-\omega}=\frac{1+i_{t}\left(1-\tau_{i}\right)}{i_{t}\left(1-\tau_{i}\right)}
$$

Como os valores de $c / y$ e $(M / p) / y$ são, respectivamente, $80 \%$ e $18 \%$, obtemos $\omega=0,99$.

A equação de Euler para o trabalho, e a expressão para o salário implicam em

$$
\frac{v_{1} v_{2} h^{v_{2-1}}}{\omega(m / p c)^{1-\omega}}=(1-\theta)(y / h)
$$

Assim como em Correia, Neves e Rebelo, assumimos $v_{2}=1,5$, que implica que a elasticidade da oferta do trabalho é igual a de um modelo que utiliza preferências com forma funcional usual. Assumindo-se que fração do tempo alocada ao mercado $h=1 / 3$, conforme o obtido por Ellery et alii (2002), obtemos $v_{1}=3,6$.

Seguindo Kanczuk (2002a), calibramos o parâmetro relativo a restrição de capital de giro, $\alpha$, calculando a diferença entre os ativos líquidos (excluindo aplicações financeiras) e os passivos líquidos (excluindo empréstimos de curto prazo) de todas as empresas listadas na BOVESPA (Bolsa de Valores de São Paulo) para os anos entre 1996 e 1999. A média ponderada (pelo tamanho da empresa) dessa diferença dividida pelas vendas totais de cada empresa é bastante estável ao longo do tempo, e tem média $\alpha=11 \%$.

Para o processo da taxa de juros,

$$
i_{t}=\left(1-\rho_{i}\right) i^{m}+\rho_{i}^{i_{t-1}}+\rho_{\pi}\left(\pi_{t-1}-\pi^{m}\right)+\rho_{y} y_{t-1}+\epsilon_{i t}
$$

em que e $\pi$ e $y$ são, respectivamente, inflação e hiato do produto, seguimos a estratégia de Taylor (1999), e utilizamos uma simples regressão OLS. Interessantemente, obtemos que o único coeficiente significativo é $\rho_{i}$, estimado em 0,58 , com "t-stat" igual a 4,3. Os coeficientes $\rho_{\pi}$ e $\rho_{y}$ são ambos insignificantes, com "pvalues" superiores a $10 \%$, e serão fixados em zero. O resíduo $\epsilon_{i}$ tem desvio padrão $\sigma_{i}=1,3 \%$.

Vale notar que uma regra de Taylor que tem o coeficiente da inflação menor do que um é freqüentemente classificada como uma política monetária passiva. Como argumenta Taylor (1999), políticas monetárias com essa característica levam a processos inflacionários explosivos em uma ampla gama de modelos. Isto, contudo, não é verdade em nosso modelo. 
Como os juros nominais seguem um processo estocástico autoregressivo, eles retornam ao seu valor médio após uma perturbação. Nesse caso, o processo inflacionário só poderia ser explosivo se os juros reais tendessem a menos um. Entretanto, no modelo aqui desenvolvido, os juros reais de longo prazo são determinados pela produtividade marginal do capital, e não podem decrescer continuamente. Assim, a inflação também não pode crescer indefinidamente. Talvez o mais surpreendente seja que a maioria dos modelos desenvolvidos por Taylor, por não terem micro-fundamentos, não satisfaça essa propriedade.

Devido à falta de uma série de horas trabalhadas, não podemos seguir a estratégia de computar os parâmetros do processo estocástico referente ao resíduo de Solow. Como alternativa, assim como Correia et alii (1995), escolhemos $\rho_{z}=0,95$, e fixamos o valor de $\sigma_{z}$ de forma a que a volatilidade da economia artificial fosse igual a da economia brasileira. Na prática, isso implicou em valores $\sigma_{z}=0,9 \%$, $\sigma_{z}=0,8 \%$, e $\sigma_{z}=0,7 \%$ para, respectivamente, as economias com $\mu=0$ e $\mu=0,25$ e $\mu=0,50$.

A seguinte tabela apresenta um sumário dos parâmetros calibrados.

\begin{tabular}{cccccccc}
\hline$\beta$ & $\theta$ & $\delta$ & $\omega$ & $v 1$ & $v 2$ & $\alpha$ & $\tau_{i}$ \\
\hline 0,98 & 0,40 & $1,7 \%$ & 0,99 & 3,6 & 1,5 & 0,11 & $39 \%$ \\
$g^{m}$ & $i^{m}$ & $\rho_{z}$ & $\sigma_{z}$ & $\rho_{i}$ & $\rho_{\pi}$ & $\rho_{y}$ & $\sigma_{i}$ \\
$2,4 \%$ & $6,7 \%$ & 0,95 & $0,8 \%$ & 0,58 & 0 & 0 & $1,3 \%$ \\
\hline
\end{tabular}

\section{Simulações e Resultados: Segundos Momentos das Séries}

As três primeiras colunas da tabela 1 apresentam os fatos estilizados da economia dos Estados Unidos (1954:1 a 2000:2) e da brasileira. Para a economia brasileira, apresentamos os valores relativos a dois intervalos: o horizonte completo (a partir de 1980), e o período somente após o Plano Real. A primeira parte da tabela 1 (tabela superior) indica a volatilidade, isto é, os desvios padrão das series. A segunda parte (tabela intermediária) mostra as correlações das series com o produto. A terceira parte (tabela inferior) corresponde à estimação da "IS dinâmica" , uma regressão do hiato do produto (i.e., da série filtrada do produto) em sua defasagem e nos juros reais. 
Tabela 1

Rigidez contratual

\begin{tabular}{|c|c|c|c|c|c|c|c|}
\hline \multicolumn{8}{|c|}{ Desvios padrão (em \%) das séries filtradas } \\
\hline Variável & EUA & Brasil & Brasil & $(1)$ & $(2)$ & $(3)$ & $(4)$ \\
\hline & 1954:01 - & 1980:01 - & 1994:03 - & $\mu=0,00$ & $\mu=0,25$ & $\mu=0,50$ & Demanda \\
\hline PIB & 1,6 & 2,7 & 1,7 & 1,8 & 1,7 & 1,7 & 1,7 \\
\hline Consumo & 0,81 & 1,9 & 1,3 & 1,1 & 1,1 & 1,4 & 2,1 \\
\hline Invest. & 5,5 & 7,7 & 5,6 & 5,1 & 4,5 & 3,9 & 0,6 \\
\hline Juros reais & 0,43 & 4,9 & 1,4 & 2,4 & 2,3 & 2,3 & 8,8 \\
\hline Juros nominais & 1,3 & 23 & 1,5 & 1,5 & 1,3 & 1,3 & 5,7 \\
\hline Inflação & 0,56 & 20 & 1,1 & 1,1 & 1,1 & 1,1 & 4,1 \\
\hline M1 (cresc.) & 0,87 & 16 & 8,9 & 0,51 & 0,52 & 0,53 & 2,0 \\
\hline Preço & 1,4 & 30 & 3,2 & 1,5 & 1,5 & 1,5 & 5,4 \\
\hline \multicolumn{8}{|c|}{ Correlação (contemporânea) com o PIB das séries filtradas } \\
\hline \multirow[t]{2}{*}{ Variável } & EUA & Brasil & Brasil & (1) & (2) & (3) & (4) \\
\hline & 1954:01 - & 1980:01 - & 1994:03 - & $\mu=0,00$ & $\mu=0,25$ & $\mu=0,50$ & Demanda \\
\hline Consumo & 0,83 & 0,93 & 0,73 & 0,99 & 0,97 & 0,94 & 1,0 \\
\hline Invest. & 0,91 & 0,89 & 0,77 & 0,99 & 0,96 & 0,87 & 0,87 \\
\hline Juros reais & $-0,23$ & $-0,29$ & $-0,09$ & 0,05 & $-0,18$ & $-0,40$ & $-0,97$ \\
\hline Juros nominais & 0,41 & $-0,21$ & $-0,33$ & 0,01 & $-0,18$ & $-0,37$ & $-0,87$ \\
\hline Inflação & 0,34 & $-0,15$ & $-0,32$ & $-0,08$ & 0,15 & 0,40 & 1,0 \\
\hline M1 (cresc.) & $-0,19$ & $-0,18$ & 0,39 & 0,06 & 0,24 & 0,43 & 0,87 \\
\hline Preço & $-0,55$ & $-0,22$ & $-0,02$ & $-0,15$ & $-0,05$ & 0,07 & 0,43 \\
\hline \multicolumn{8}{|c|}{ IS dinâmica: variável dependente $=$ hiato } \\
\hline \multirow[t]{2}{*}{ Coeficiente } & EUA & Brasil & Brasil & $(1)$ & $(2)$ & (3) & (4) \\
\hline & 1954:01 - & 1980:01 - & 1994:03 - & $\mu=0,00$ & $\mu=0,25$ & $\mu=0,50$ & Demanda \\
\hline Constante & $\begin{array}{c}0,00 \\
(0,00)\end{array}$ & $\begin{array}{c}0,00 \\
(0,00)\end{array}$ & $\begin{array}{c}0,15 \\
(0,18)\end{array}$ & 0,00 & 0,00 & 0,01 & 0,01 \\
\hline Hiato $(-1)$ & $\begin{array}{c}0,85 \\
(0,05)\end{array}$ & $\begin{array}{c}0,68 \\
(0,08)\end{array}$ & $\begin{array}{c}0,37 \\
(0,17)\end{array}$ & 0,66 & 0,57 & 0,39 & $-0,23$ \\
\hline Juros reais & $\begin{array}{l}-0,09 \\
(0,12)\end{array}$ & $\begin{array}{l}-0,12 \\
(0,04)\end{array}$ & $\begin{array}{l}-0,14 \\
(0,17)\end{array}$ & 0,02 & $-0,10$ & $-0,23$ & $-0,17$ \\
\hline
\end{tabular}

Desvios padrão entre parênteses.

Note que o lado real das economias, isto é, as séries de produto, consumo, investimento e juros reais são bem semelhantes quando comparadas entre si. $\mathrm{O}$ consumo tem cerca de $80 \%$ da volatilidade do produto, enquanto o investimento é aproximadamente três vezes mais volátil. O consumo e o investimento são fortemente pro-cíclicos, enquanto os juros reais são contra-cíclicos. E a IS dinâmica apresenta clara influência (negativa) dos juros sobre o produto. A diferença entre as economias parece estar na magnitude das volatilidades de todas as suas séries. O Brasil a partir de 1980 é mais volátil que o Brasil pós-Real, que é mais volátil que os Estados Unidos.

A mesma semelhança não ocorre quando observamos as variáveis nominais. De fato, como Backus e Kehoe (1992) e Gavin e Kydland (1999) observam, os segundos momentos das variáveis nominais não parecem ser robustos a diferentes intervalos de tempo. Enquanto nos EUA a inflação e os juros nominais são procíclicos, no 
Brasil eles são contra-cíclicos. ${ }^{2}$ O crescimento do agregado monetário M1 muda de contra-cíclico para pro-cíclico conforme escolhemos o período de amostra para a economia brasileira. O mesmo ocorre com o índice de preço, que no período mais recente tem correlação nula com o produto.

As seguintes três colunas da tabela 1 mostram os resultados das simulações com nossas economias artificiais. As economias de (1) a (3) são definidas por seus diferentes graus de rigidez de contratos de trabalho, $\mu$, isto é, a fração de firmas que paga salários fixados com um período de avanço.

Os efeitos de aumentos dessa rigidez nos segundos momentos das séries parecem ser "monotônicos". Mais rigidez implica em maiores volatilidades do produto e do consumo e menor volatilidade do investimento. Mais rigidez faz com que os juros nominais e reais se tornem mais contra-cíclicos, enquanto a inflação, o crescimento de M1 e o preço se tornem mais pró-cíclicos. Mais rigidez implica em uma IS dinâmica em que o coeficiente dos juros tem maior módulo.

A intuição para esse resultado reside no fato de que um maior grau de rigidez de preços faz com que os choques monetários se tornem (relativamente) mais importantes. Como parte dos salários nominais está fixa, um aumento inesperado na quantidade de moeda faz com que a demanda por trabalho se eleve, enquanto as famílias são obrigadas a ofertar a quantidade de trabalho que equilibra o mercado, aumentando sua produção. Dessa forma, choques monetários implicam em volatilidade de produto sem contrapartida no investimento, já que a tecnologia (produtividade) está inalterada. Pela mesma lógica, aumentam as correlações da inflação e dos preços com o produto.

Por outro lado, como que a restrição de capital de giro se torne quantitativamente mais importante (devido a maior volatilidade dos juros), choques monetários aumentam o efeito dos juros nominais sobre a demanda por trabalho, e assim sobre o produto. Isto aumenta (em módulo) a correlação entre os juros nominais e o produto. Como parte dos preços está fixa, o mesmo vale para a correlação entre os juros reais e o produto, e para o coeficiente dos juros reais na IS dinâmica.

Uma observação da tabela 1 revela que não é fácil escolher a economia artificial com melhor performance. As três economias têm correlações e volatilidades do produto, consumo e investimento relativamente próximas às dos dados. A economia (1) gera séries de inflação contra-cíclica, como os dados, mas apresenta têm juros pró-cíclicos, em contraste com a realidade. As economias (2) e (3) têm o problema inverso: os juros são contra-cíclicos, mas a inflação é pró-cíclica.

Seguindo a praxe dos estudos de ciclos reais, neste artigo não escolhemos nenhu-

\footnotetext{
${ }^{2}$ Kydland and Prescott (1991) questionam a relação pro-cíclica entre as variáveis nominais e reais, para a economia dos Estados Unidos.
} 
ma medida formal de performance relativa das economias artificiais. Ao invés, optamos por sugerir qual é nossa melhor economia através de uma observação conjunta de todos os segundos momentos, e de nosso conhecimento prévio sobre a robustez desses valores nos dados reais. No nosso caso em particular, sabemos que as variáveis do lado real apresentam maior robustez que as variáveis nominais. Além disso, sabemos que a IS dinâmica é uma das únicas relações que pode ser robustamente estimada para diferentes períodos da economia brasileira. Com isso em mente, propomos que a economia (2), com rigidez de $25 \%$, é nossa melhor economia. Nela, a correlação entre os juros e o produto é claramente negativa, e os coeficientes na IS dinâmica têm magnitude próxima à estimada.

Nas próximas seções reportarmos somente simulações realizadas com economias em que $25 \%$ dos salários são fixados anteriormente. Mas vale mencionar que os resultados qualitativos não se alteram, caso sejam utilizados $\mu=0$ e $\mu=50 \%$.

\section{Modelo Estrutural e Choques de Oferta}

Conforme apresentam Taylor (1999) e Bogdanski et alii (2001), entre outros, os modelos estruturais para simulações de metas inflacionárias são tipicamente compostos de três equações:

$$
\begin{gathered}
i_{t}=\left(1-\rho_{i}\right) i_{t-1}+\rho_{\pi} \pi_{t-1}+\rho_{y} y_{t-1}+\epsilon_{i t} \\
y_{t}=\varphi_{y} y_{t-1}+\varphi r_{t}+\epsilon_{y t} \\
\pi_{t}=\lambda_{\pi} \pi_{t-1}+\lambda_{e} e_{t}+\lambda_{y} y_{t-1}+\epsilon_{\pi t}
\end{gathered}
$$

em que $e_{t}$ corresponde à depreciação, isto é, a variação do preço (em reais) do dólar. Note que já utilizamos as duas primeiras equações, quais sejam, a regra monetária (taxa de juros) e a "IS dinâmica". A terceira, chamada de "Phillips" se torna agora o objeto principal da nossa atenção.

A história da curva de Phillips é longa e conhecida, talvez o surpreendente seja o fato dela atualmente estar presente em virtualmente todo trabalho de metas inflacionárias, após ter sido tão fortemente criticada. Bogdanski et alii (2001), por exemplo, motivam essa curva através de contratos de trabalhos, de forma bem semelhante à proposta aqui.

Para este trabalho, o item importante é o fato da curva de Phillips ter uma motivação econômica baseada exclusivamente em choques de demanda. Sua lógica sempre é a de que um choque de demanda faz com que o produto fique acima do 
produto potencial, o que implica em um aumento da inflação. Isso explica porque o coeficiente $\lambda_{y}$ deve ser positivo.

O problema é que por "produto acima do produto potencial" entende-se, simplesmente, que o produto filtrado pelo filtro H-P (ou por uma tendência geométrica) tem valor positivo. Um hipotético choque de oferta também implica em que o produto filtrado seja positivo, embora signifique que houve um aumento do produto potencial. O econometrista, é claro, não pode saber o que foi que ocorreu. Se houve um choque de oferta, a estimação busca um coeficiente positivo para $\lambda_{y}$, enquanto este talvez não o seja. Como há tanto choques de oferta como de demanda, o coeficiente estimado fica viesado.

O mesmo problema pode ser colocado de forma mais formal. Quando se estima a curva de Phillips se assume que o resíduo $\epsilon_{\pi t}$ tem correlação nula com o regressor $y_{t-1}$. Mas choques de oferta afetam a ambos, tornando sua correlação não nula.

Vale notar que esta mesma observação sobre a dificuldade de medição do produto potencial já foi apontada por Woodford (2001), num contexto distinto do presente: "em teoria, uma grande variedade de choques reais afetam o produto potencial, ..., estes incluem choques tecnológicos, mudanças na oferta de trabalho, variações no consumo do governo, variações na impaciência das famílias, e variações na produtividade das oportunidades de investimento disponíveis, e não há nenhuma razão para assumir que todos esses fatores seguem tendências suaves (tradução nossa)". Após sugerir que o produto potencial deve estar (positivamente) relacionado com o custo do trabalho unitário, Woodford mostra que este apresenta correlação negativa com o PIB sem tendência. Isto é, a medida usual do produto potencial parece ter movimentos na direção contrária ao que a teoria sugere.

\section{Pseudo-Dólar}

Nossas simulações geram séries de dados que nos permitem estimar a IS dinâmica. Mas para que possamos utilizar nossa economia para estimar o modelo estrutural completo, incluindo curva de Phillips, é necessário que obtenhamos uma série de depreciação cambial. Com esse objetivo, propomos aqui a derivação de um "pseudo-dólar" . Entretanto, para isso, é mandatório um importante caveat.

O modelo até agora exposto apresenta micro-fundamentos para quase todos os seus componentes. As única hipóteses completamente ad-hoc são a regra de Taylor e o esquema de contratos de salário que, em nossa opinião, não tem bom respaldo teórico. Esta seção, contudo, propõe uma derivação de um preço através de uma motivação bem menos fundamentada. Para deixar este ponto mais explícito, vale 
dizer que no modelo há, para cada período, cinco preços correspondentes a cinco bens: numerário (moeda), salário flexível (trabalho), salário fixo (trabalho contratado), juros (capital) e preço final (consumo, investimento e produto). Esta observação, em si, já deixa claro que não há espaço na modelagem para formalmente obter o preço do dólar. Mas talvez o que mais chame atenção é o fato de nossa economia ser fechada. Seja qual for a crítica preferida, ela é bem recebida. Entretanto, fortunadamente, essa crítica só se aplica às estimações da curva de Phillips, e mesmo sem estas a lógica geral de nossos resultados ainda vale.

A derivação que propomos é baseada no trabalho de Dornbusch (1976), o qual talvez constitui o modelo mais utilizado na macroeconomia economia aberta tradicional. O apelo econômico é que dólar é um preço mais flexível que os demais, e assim se ajusta primeiro quando de um choque monetário. Em especial, isto faz com que, quando há uma emissão de moeda, o câmbio apresente o conhecido "overshoot".

No nosso caso, contamos com a existência de dois mercados de trabalho, um deles paga salários que foram contratados no período anterior. Nossa proposta é então utilizar a relação entre os salários para balizar a relação entre o dólar e o preço final.

Para formalizar essa intuição, assumimos que o salário fixo e o flexível seguem as relações,

$$
\begin{aligned}
W & =(\text { dolar })^{\mu 1} p^{1-\mu 1} \\
W^{c} & =(\text { dolar })^{\mu 2} p^{1-\mu 2}
\end{aligned}
$$

isto é, os salários são uma combinação do preço com o pseudo dólar, $\mu 1$ e $\mu 2$ são parâmetros. Evidentemente, se entendemos que o dólar é mais flexível que o preço como um todo, que $\mu 1>\mu 2$. As expressões acima implicam em

$$
(\operatorname{dolar} / p)=\left(W / W^{c}\right)^{\mu 1 \mu 2}
$$

Como não sabemos os valores de $\mu 1$ ou $\mu 2$, calibramos $(\mu 1-\mu 2)$ diretamente. Em especial, escolhemos $(\mu 1-\mu 2)$ de forma que o coeficiente da desvalorização na curva de Phillips, $\lambda_{e}$, seja igual ao estimado. Essa metodologia tem o tradicional problema de utilizar-se de informações dos segundos momentos das séries, mas no caso particular não temos nenhum microfundamento para uma calibração "correta".

Para uma melhor intuição das implicações das hipóteses realizadas, construímos as respostas a impulso a um choque monetário. As figuras 1 e 2, respec- 
tivamente, mostram o perfil das variáveis reais e nominais conseqüentes de uma redução (exógena) dos juros nominais. As variáveis reais estão logaritmadas, e expressas em torno de suas médias Note que o produto e o consumo aumentam mais que o investimento. Isto ocorre porque não houve uma melhora na tecnologia e na produtividade marginal do capital.

Figura 1

Variáveis reais - choque monetário

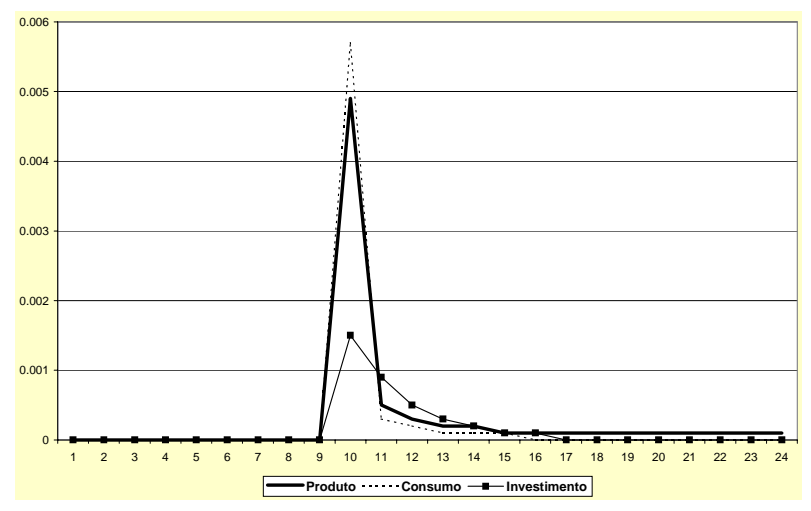

Figura 2

Variáveis nominais - choque monetário

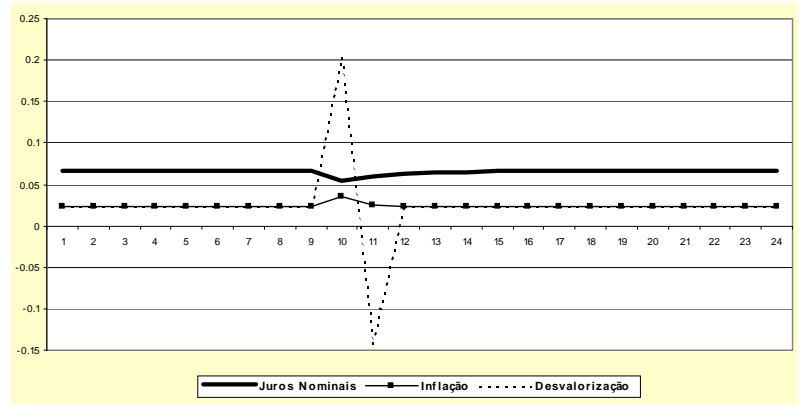

A emissão de moeda conseqüente da redução dos juros implica em aumento da inflação, que dura poucos períodos. A desvalorização cambial é muito mais expressiva que a inflação no primeiro período, seus valores são respectivamente $20 \%$ e 3,5\%. No segundo trimestre após o choque a desvalorização torna-se negativa, mostrando que o dólar retorna ao preço médio da economia, após o overshoot. 


\section{Simulações e Resultados: Modelo Estrutural}

A tabela 2 mostra as estimações do modelo estrutural realizadas para os EUA, para o Brasil, e sobre os dados gerados por nossas economias artificiais. Todas as colunas referem-se a economias com $25 \%$ de rigidez. A primeira coluna corresponde à mesma economia (2) da primeira tabela, em que há choques tanto de oferta como de demanda. As colunas seguintes mostram economias em que há somente choques de demanda (economia (4)) ou somente choques de oferta (economia (5)).

Tabela 2

Estimações do modelo estrutural

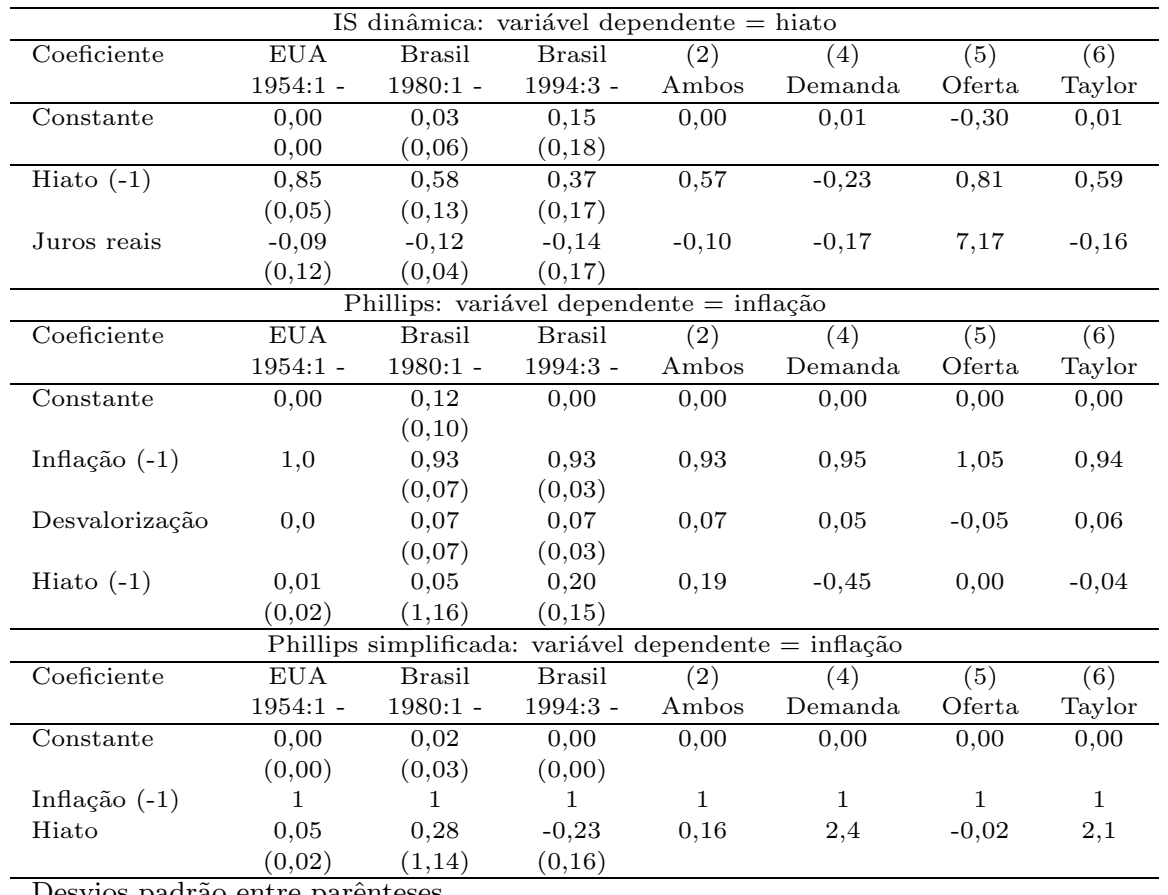

Desvios padrão entre parênteses

As primeiras linhas referem-se a estimação da "IS dinâmica", repetidas da tabela 1. A seguir estão indicadas as estimações da curva de Phillips que inclui a desvalorização obtida através do "pseudo-dolar" . Seguindo o procedimento típico do BACEN, estas estimações são feitas com a hipótese de que os coeficientes da inflação defasada e da desvalorização somam um, i.e., $\lambda_{\pi}+\lambda_{e}=1$.

As últimas três linhas mostram uma estimação de uma "curva de Phillips simplificada", em que não há um termo de desvalorização, o hiato não está defasado, 
e o termo para a inflação defasada é por hipótese igual a um. Esta especificação simplificada está mais em linha com a IS, e tem a vantagem de não depender do pseudo-dolar sem micro-fundamentos. Por outro lado, não corresponde a especificação utilizada pelo BACEN, talvez porque suas estimações sejam contraintuitivas.

A IS dinâmica é robustamente estimada para diferentes períodos da economia brasileira. Para os EUA, o coeficiente dos juros reais não pode ser estimado com precisão, mas seu valor esperado é surpreendentemente próximo ao brasileiro. A estimação para a economia com ambos os choques está dentro do intervalo de confiança, e muito próxima à do período pós 1980. A estimação somente com choques de demanda tem coeficiente dos juros que também se encontra no intervalo de confiança, embora com maior módulo. A estimação somente com choques de oferta é diametralmente oposta, indicando uma relação positiva entre juros e hiato. Isto ocorre porque choques de oferta representam um aumento da produtividade marginal do capital, e assim dos juros reais.

Antes de partir para a análise da curva de Phillips vale a pena refletir sobre a possibilidade de que a economia brasileira só apresente choques de demanda. Esta seria uma proposta rapidamente rejeitada por pesquisadores da área de ciclos reais, mas está de acordo com a intuição de alguns economistas "novos-keynesianos". Para este artigo, este questionamento torna-se particularmente relevante porque se relaciona com o nosso argumento sobre a identificação da curva de Phillips. Se a economia só apresenta choques de demanda, a curva de Phillips não sofre do problema de identificação que acreditamos sofrer.

O fato da economia somente com choques de demanda (economia (4)) implicar em uma IS em que o coeficiente para o hiato defasado é negativo oferece um argumento contrário inicial. Mas para uma melhor análise, na última coluna da tabela 1 colocamos todos os segundos momentos dessa economia. Note que ela segue a mesma tendência observada quando de um aumento de rigidez, isto é, a economia só com choques de demanda corresponderia a uma economia com um grau de rigidez bastante superior a 50\%. Note que, no que tange as variáveis reais, as quais apresentam bastante robustez em estudos internacionais, a economia (4) tem performance bastante ruim. A série de investimentos tem volatilidade bem inferior ao PIB (cerca de um terço), enquanto os dados reais, para vários países e intervalos de tempo, têm uma série de investimento que é cerca de três vezes mais volátil que o PIB. Isto se deve, conforme discutido na seção anterior (figura 1), a excessiva estabilidade do produto marginal do capital.

Curiosamente, o fato de uma economia somente com choques de demanda ter performance tão ruim parece estar de acordo com o ponto levantado por Woodford 
(2001). Conforme já citamos, Woodford obtém que o produto potencial medido usualmente tem correlação negativa com aquele sugerido pela teoria. Isto seria uma conseqüência natural de economias em que os choques de oferta são mais importantes quantitativamente do que os de demanda, que é o que os nossos resultados sugerem.

Voltando ao modelo estrutural, note que a estimação da curva de Phillips varia com o período em questão. O coeficiente do hiato é fortemente rejeitado para o período completo, e mesmo para o período pós-plano Real tem p-value igual a 17\%. No caso da Phillips simplificada, esse coeficiente é novamente insignificante no período completo, e seu sinal é revertido para o período recente. Talvez o interessante é que esse coeficiente se torna relevante para a economia dos EUA.

Nossa economia artificial com ambos os choques apresenta uma curva de Phillips bastante próxima à estimada, mas dois comentários são devidos. Primeiro, o coeficiente da desvalorização não deve ser tomado como um triunfo do modelo, já que o parâmetro $\xi$ foi calibrado para que seu valor fosse igual ao estimado com os dados reais. Em segundo lugar, esta estimação não é robusta a pequenas variações nos parâmetros calibrados. Em especial, alterando-se o valor de $v_{2}$, que é o parâmetro sobre o qual temos maior incerteza, a estimação da Phillips altera-se bastante. Para $v_{2}=1,1$, por exemplo, o coeficiente do hiato é estimado em 0,06.

A estimação da curva de Phillips para a economia somente com choques de oferta indica um coeficiente do hiato igual a zero. Isto se deve ao fato de choques de oferta alterarem o PIB potencial, não sendo inflacionários, conforme sugere a intuição econômica. Para a economia somente com choques de demanda, o coeficiente se torna negativo. Não temos uma boa intuição para isso. Nossa intuição seria a de que o coeficiente se tornasse com maior módulo, mas se mantivesse positivo. Acreditamos que esse resultado é conseqüência do regressor desvalorização, que captura totalmente os efeitos desses choques.

A estimação da Phillips simplificada é mais intuitiva. A economia com choques de demanda apresenta coeficiente do hiato positivo e com grande magnitude. E a economia com choques de oferta tem coeficiente do hiato praticamente nulo. Mas, como já mencionado, a estimação para o Brasil pós Real é contra intuitiva, talvez mesmo por não ser robusta.

O mais importante é que para a IS e qualquer uma das curvas de Phillips as estimações são bastante diferentes quando consideramos a presença dos choques de oferta. Que choques de oferta são necessários para que a economia artificial se comporte como a real. E que, conseqüentemente, o problema de identificação das estimações usuais do modelo estrutural é quantitativamente importante.

$\mathrm{Na}$ última coluna da tabela 2 apresentamos as estimações do modelo estrutural 
para uma economia sujeita a uma regra monetária diferente da observada para o Brasil. Escolhemos, arbitrariamente, a regra proposta por Taylor (1999), em que o processo para a taxa de juros tem coeficientes para a inflação e para o produto de respectivamente $\lambda_{\pi}=1,5$ e $\lambda_{y}=0,5$, e coeficiente para a defasagem dos juros igual a zero $\left(\lambda_{i}=0\right)$. Esta regra tem a propriedade de apresentar uma performance bastante boa em diferentes modelos, e parece aproximar a política observada nos EUA.

Estimar o modelo em uma economia artificial calibrada para o Brasil, mas em que a política monetária segue essa regra constitui um teste para a relevância da Crítica de Lucas. Se os resultados forem sensivelmente diferentes dos obtidos anteriormente, para a política em vigor, é porque a reação dos agentes econômicos a novas regras é quantitativamente relevante. E isso é exatamente o que os nossos resultados sugerem. Nossa economia (6) apresenta uma IS dinâmica bastante próxima a economia (2), mas a curva de Phillips tem coeficiente do hiato negativo. Se o nosso modelo estiver correto, e o BACEN alterar sua política seguindo a recomendação de Taylor, as estimações do modelo estrutural vão se alterar, a curva de Phillips estimada vai se tornar contra intuitiva, e, quem sabe, o BACEN vai terminar por abandoná-la.

\section{Conclusões}

Modelos microfundamentados podem ser implementados para a economia brasileira, e seriam utilizados como ferramenta de auxílio para a tomada de decisão de política monetária. Por eles serem robustos a Crítica de Lucas, ao menos potencialmente, muitos pesquisadores acreditam que eles naturalmente deverão substituir os modelos atualmente utilizados, que são baseados em estimações econométricas de formas reduzidas.

O presente artigo não somente sugere que isto é verdade, mas também aponta um outro problema com as estimações usuais. Por não efetivamente considerarem a presença de choques de oferta, essas estimações são viesadas, como em qualquer problema de identificação. Um modelo com microfundamentos não está sujeito a essa falha, já que os choques de oferta e demanda podem ser especificados separadamente, através da teoria.

Nossos experimentos indicam que esse problema na estimação dos modelos estruturais é quantitativamente relevante. Na prática, isso significa que simulações realizadas sobre esses modelos podem levar a conclusões erradas, e implicar em erros na tomada de decisão de política monetária.

Adicionalmente, nossos resultados sugerem que a Crítica de Lucas também é 
quantitivamente importante. Isto significa que, mesmo que o problema de identificação fosse de alguma forma resolvido, modelos sem microfundamentos não são indicados para realização de política, por não considerarem a reação dos agentes econômicos. Novamente, os resultados de simulação seriam equivocados, já que os coeficientes se alterariam no momento em que a política fosse modificada.

Há, contudo, um longo caminho a ser percorrido pelos modelos microfundamentados. Nesse artigo obtivemos o valor da depreciação cambial, necessária para a estimação da curva de Phillips, de forma ad-hoc e não micro-fundamentada. Além disso, como é usual na literatura, assumimos rigidez salarial sem uma justificativa adequada. E ainda assim, obtivemos dados simulados que não estão completamente em linha com os dados reais.

\section{Referências}

Araujo, C. H. V. \& Ferreira, P. C. G. (1999). Reforma tributária, efeitos alocativos e impactos de bem-estar. Revista Brasileira de Economia, 53(2):133-166.

Backus, D. C. \& Kehoe, P. (1992). International evidence on the historical properties of business cycles. American Economic Review, 82.

Blinder, A. S. \& Mankiw, N. G. (1984). Aggregation and stabilization policy in a multi-contract economy. Journal of Monetary Economics, 13:67-83.

Bogdanski, J., Springer, P. F., Goldfajn, I., \& Tombini, A. A. (2001). Inflation targeting in Brazil: Shocks, backward-looking prices and IMF conditionality. Working Paper Series 24, BACEN.

Chari, V. V., Kehoe, P. J., \& McGrattan, E. R. (2000). Can sticky prices generate volatile and persistent real exchange rates? NBER Working Paper, 7869.

Christiano, L. J. \& Eichenbaum, M. (1995). Liquidity effects, monetary policy, and the business cycle. Journal of Money, Credit, and Banking, 27(4):1113-1136.

Christiano, L. J., Eichenbaum, M., \& Evans, C. L. (1997). Sticky price and limited participation models of money: A comparison. European Economic Review, 41:1201-1249.

Christiano, L. J. \& Gust, C. J. (2000). Taylor rules in a limited participation model. NBER Working Paper 7017. 
Cooley, T. F. (1995). Frontiers of Business Cycle Research. Princeton University Press.

Cooley, T. F. \& Hansen, G. D. (1998). The role of monetary shocks in equilibrium business cycle theory: Three examples. European Economic Review, 42:605-17.

Correia, I., Neves, J., \& Rebelo, S. (1995). Business cycles in a small open economy. European Economic Review, 39:1089-1113.

Correia, I. \& Teles, P. (2000). Optimal inflation tax. Review of Economic Dynamics, 2(2):325-46.

Dornbusch, R. (1976). Expectations and exchange rate dynamics. Journal of Political Economy, 84(6):1161-76.

Ellery, R. J., Gomes, V., \& Sachida, A. (2002). Business cycle fluctuations in Brazil. Revista Brasileira de Economia, 56(2).

Feldstein, M. (1999). Comment on: Interest rate rules in an estimated sticky price model. In Taylor, J. B., editor, Monetary Policy Rules.

Floden, M. (2000). Endogenous monetary policy and the business cycle. European Economic Review, 44:1409-1429.

Fraga, A. (2000). Regimes cambiais e monetários. Seminários Dimac No. 6, IPEA.

Gavin, W. T. \& Kydland, F. E. (1999). Endogenous money supply and the business cycle. Review of Economic Dynamics, 2(2):347-69.

Gollin, D. (2002). Getting income shares right. Journal of Political Economy, $110(2): 458-74$.

Greenwood, J., Hercowitz, Z., \& Huffman, G. (1988). Investment, capacity utilization and the business cycle. American Economic Review, 78:402-417.

Kanczuk, F. (2001). Business cycles in a small open brazilian economy. Economia Aplicada, 5(3):455-71.

Kanczuk, F. (2002a). Juros reais e ciclos e ciclos reais brasileiros. Revista Brasileira de Economia, 56(2):249-68.

Kanczuk, F. (2002b). Usando ciclos reais para construir cenários macroeconômicos. Manuscrito, Versão Preliminar Circulou Com Título "Usando Ciclos Reais Para Projetar Tendência" , Anais Da ANPEC 2001. 
Kanczuk, F. \& Faria, F. J. (2000). Ciclos reais para a indústria brasileira. Estudos Econômicos, 30(3):335-350.

Kydland, F. \& Prescott, E. C. (1990). Business cycle: Real facts and a monetary myth. Federal Reserve of Minneapolis Quarterly Review, 14(2).

Lucas, R. E. J. (1976). Econometric policy evaluation: A critique. Carnegie Rochester Series on Public Policy.

Taylor, J. B. (1999). Monetary Policy Rules. University of Chicago Press.

Woodford, M. (2001). The taylor rule and optimal monetary policy. American Economic Review. 